\title{
Multi-Phase Dynamics of Magnetized Interstellar Medium
}

\author{
Shu-ichiro Inutsuka \\ Department of Physics, Nagoya University, Chikusa-ku, Nagoya, Aichi 464-8602, Japan \\ email: inutsuka@nagoya-u.jp
}

\begin{abstract}
The recent progress in our understanding of the dynamics of muliti-phase interstellar medium (ISM) is reviewed. Non-linear perturbations (e.g., shock waves or time-dependent radiation field) lead to the interchange between warm phase and cold phase via thermal instability. Dynamical modelling of this phase transition dynamics is essential in describing ubiquitous turbulence in ISM and the formation of molecular clouds. A concept of magnetically multi-phase medium is introduced. Recent finding of the magnetic field amplification in the blast wave propagating in magnetized multi-phase ISM is providing a strong motivation for rapid acceleration of cosmic rays.
\end{abstract}

Our understanding on the physical processes in the transition between warm neutral medium (WNM) and cold neutral medium (CNM) is substantially increased in the last decade. The basic property of thermal instability (hereafter, TI; Field 1965) and resultant phase transition dynamics of interstellar medium (ISM) is reviewed in, e.g., Inutsuka et al. (2005), Hennebelle et al. (2007). When the ISM is swept-up by a shock wave, the gas temperature in the shock-compressed gas first increases by shock-heating but eventually decreases because of higher cooling rate in dense gas. During gas temperature is in the range from $\sim 6,000 \mathrm{~K}$ to $\sim 300 \mathrm{~K}$, the gas is subject to thermal instability. The multi-dimensional nonlinear evolution of TI has been first studied by Koyama \& Inutsuka (2002). They have found that TI generates supersonic turbulence that does not decay as long as the shock wave continues its propagation (see also, Audit \& Hennebelle 2005; Hennebelle \& Audit 2007; Koyama \& Inutsuka 2000, 2004; Inutsuka \& Koyama 2002, 2004, 2007; Inoue et al. 2006, 2007, 2008, 2009; Nagashima et al. 2005, 2006; Yamada et al. 2007; Heitsch et al.2005; Gazol et al. 2005; Vazquez-Semadeni et al.2006, TI in the shock propagating model is continously happening in "fresh" thermally unstable gas that is continuously provided by shock compression and heating. The motion of CNM that is supersonic with respect to the CNM sound speed but subsonic to WNM can survive shock dissipation in surrounding WNM.

Using two-dimensional two-fluid magnetohydrodynamic simulations Inoue \& Inutsuka (2008, 2009) have studied the converging supersonic flows of magnetized warm neutral medium that generate a shocked slab of thermally unstable gas in which cold clouds form, and shown that the effect of magnetic field on the phase transition dynamics is crucial. They found that in the shocked slab magnetic pressure dominates thermal pressure and the thermal instability grows in the isochorically cooling, thermally unstable slab that leads to the formation of HI clouds whose number density is typically $n \lesssim 10^{2} \mathrm{~cm}^{3}$, even if the angle between magnetic field and converging flows is small. They also found that even if there is a large dispersion of magnetic field, evolution of the shocked slab is essentially determined by the angle between the mean magnetic field and converging flows. Thus, the direct formation of molecular clouds by piling up warm neutral medium does not seem to be a typical molecular cloud formation process, unless the direction of supersonic converging flows is biased to the orientation of mean magnetic field by some mechanism. However, when the angle is small, the H I shell generated as a result of converging flows is massive and possibly evolves to molecular clouds, provided gas in the massive H I shell is piled up again along the magnetic field line. Thus, another subsequent shock wave can again pile up the gas of the massive shell and produce a larger cloud. We thus emphasize the importance of multiple episodes of converging flows, as a typical formation process of molecular clouds. A natural consequence of this complicated history of molecular clouds is highly non-uniform spatial distribution of the ratio of magnetic pressure to gas pressure. In such 
configurations, large gas pressure gradients can be partially balanced by the magnetic pressure gradient, thus, maintaining high density contrast in dense clouds. More detailed analyses on the physical property of this magnetically multi-phase medium is clearly needed to understand the realistic evolution of ISM.

Since molecular clouds form through the contraction of HI gas, the question arises as to whether this structure is maintained in the molecular phase or not. Recently Hennebelle \& Inutsuka (2006) investigated whether the warm neutral atomic hydrogen (WNM) can exist in molecular clouds. They considered the dissipation of MHD waves propagating in the WNM inside the cloud, and found that it is sufficient to allow the existence of WNM inside a molecular cloud of size $\simeq 1 \mathrm{pc}$ having pressure equal to $\simeq 10 \times P_{\text {ISM }}$. This result suggests the possibility that channels of magnetized WMN may provide efficient energy injection for sustaining internal turbulence which otherwise decays in a crossing time.

Inoue, et al. (2009) examined magnetohydrodynamic simulations of the propagation of a strong shock wave (e.g., due to a young supernova remnant) through the interstellar two-phase medium composed of small-scale cloudlets and diffuse warm neutral medium. They have shown that the shock-compressed shell becomes turbulent owing to the preshock density inhomogeneity, and magnetic field amplification takes place in the shell. The maximum field strength is determined by the energy equi-partition condition in the post-shock region, which gives the field strength on the order of $1 \mathrm{mG}$ in the case of shock velocity $\sim 10^{3} \mathrm{kms}^{-1}$. This strongly magnetized turbulent region can be an ideal site for cosmic ray acceleration, and agrees with the spatial scale of time-dependent X-ray hot spots recently observed in young supernova remnants (Uchiyama et al. 2007).

\section{References}

Audit, E. \& Hennebelle, P. 2005, A\&A, 433, 1

Field, G. B. 1965, ApJ, 142, 531

Gazol, A. Vazquez-Semadeni, E., \& Kim, J. 2005, ApJ 630, 911

Heitsch, F. et al. 2005, ApJ, 633, L113

Hennebelle, P. \& Audit, E. 2007 A\&A 465, 431

Hennebelle, P. \& Inutsuka, S. 2006 ApJ 647, 404

Hennebelle, P., Mac Low, M.-M., \& Vazquez-Semadeni, E. 2008 "Structure formation in the universe", Ed. G. Chabrier (Cambridge University Press)

Inoue, T. \& Inutsuka, S. 2008, ApJ 687, 303 ; 2009, ApJ 704, 161

Inoue, T., Inutsuka, S., \& Koyama, H. 2006, ApJ 652, 1131 ; 2007, ApJ 658, L99

Inoue, T. Yamazaki, R., \& Inutsuka, S. 2009, ApJ 695, 825

Inutsuka, S., \& Koyama, H. 2002, ApSS, 281, 67; 2004, RMxAC 22, 26; 2007, ASPCS, 365, 162;

Inutsuka, S., Koyama, H., \& Inoue, T. 2005, AIPCP 784, 318

Koyama, H. \& Inutsuka, S. 2000, ApJ, 532, 980 ; 2002, ApJ 564, L97 ; 2004, ApJ 602, L25

Nagashima, M., Koyama, H., \& Inutsuka, S. 2005, MN, 361, L25 ; 2006, ApJL 652, L4

Uchiyama, Y., et al. 2007, Nature, 449, 576

Vazquez-Semadeni, E., et al. 2006, ApJ, 643, 245

Yamada, M., Koyama, H., Omukai, K., \& Inutsuka, S. 2007, ApJ 657, 849 